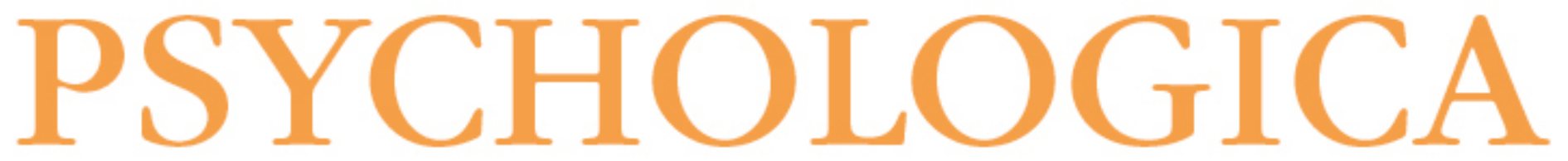

\title{
Entrapment: conceito, definição e características psicométricas da versão portuguesa da Escala de Entrapment
}

Autor(es): $\quad$ Carvalho, Serafim; Gouveia, José Pinto; Castilho, Paula; Pimentel, Paulo

Publicado por: Imprensa da Universidade de Coimbra

URL

persistente: URI:http://hdl.handle.net/10316.2/5584

DOI: $\quad$ DOI:http://dx.doi.org/10.14195/1647-8606_54_15

Accessed : $\quad$ 26-Apr-2023 16:30:56

A navegação consulta e descarregamento dos títulos inseridos nas Bibliotecas Digitais UC Digitalis, UC Pombalina e UC Impactum, pressupõem a aceitação plena e sem reservas dos Termos e Condições de Uso destas Bibliotecas Digitais, disponíveis em https://digitalis.uc.pt/pt-pt/termos.

Conforme exposto nos referidos Termos e Condições de Uso, o descarregamento de títulos de acesso restrito requer uma licença válida de autorização devendo o utilizador aceder ao(s) documento(s) a partir de um endereço de IP da instituição detentora da supramencionada licença.

Ao utilizador é apenas permitido o descarregamento para uso pessoal, pelo que o emprego do(s) título(s) descarregado(s) para outro fim, designadamente comercial, carece de autorização do respetivo autor ou editor da obra.

Na medida em que todas as obras da UC Digitalis se encontram protegidas pelo Código do Direito de Autor e Direitos Conexos e demais legislação aplicável, toda a cópia, parcial ou total, deste documento, nos casos em que é legalmente admitida, deverá conter ou fazer-se acompanhar por este aviso. 


\section{NÚMERO 54}

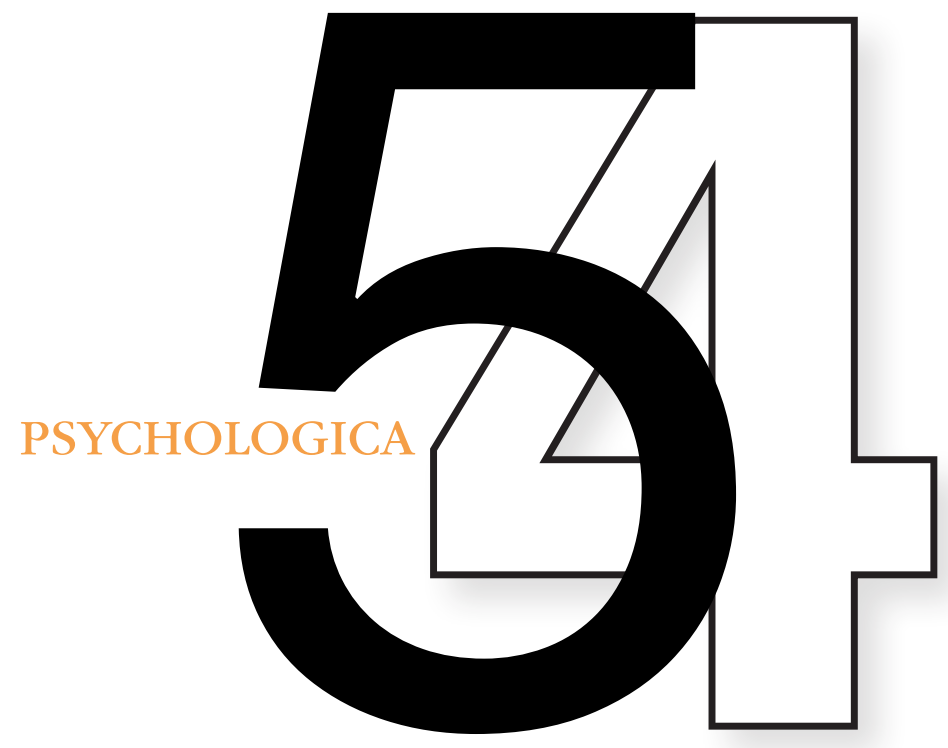

AVALIAÇÃO PSICOLÓGICA EM CONTEXTO CLÍNICO 


\title{
Entrapment - conceito, definição e características psicométricas da versão portuguesa da Escala de Entrapment
}

\author{
Serafim Carvalho',2, José Pinto-Gouveia², Paula Castilho², Paulo Pimentel3,4
}

O papel das estratégias evolucionárias na psicopatologia vem sendo amplamente estudado nos últimos anos, particularmente os mecanismos de luta e fuga. $\mathrm{O}$ entrapment designa a inibição da estratégia de fuga quando esta está psicobiologicamente activada. Contextos de vida com características de entrapment estão ligados a stress, ansiedade, depressão e suicídio.

Neste trabalho apresentam-se as características psicométricas da Escala de Entrapment (EE) originalmente desenvolvida por Gilbert \& Allan (1998); é composta por duas escalas, a escala de Entrapment Interno (EEI) relacionada com activação para a fuga motivada por estados emocionais aversivos e a escala de Entrapment Externo (EEE), quando o desejo de fuga é estimulado por acontecimentos externos. A escala foi avaliada em três amostras: estudantes $(n=608)$, população geral $(n=406)$ e doentes deprimidos $(n=106)$.

A escala reproduziu os resultados dos seus autores. Demonstrou uma elevada consistência interna, com $\alpha$ de Cronbach oscilando entre .85 e .93; uma estrutura unidimensional para cada escala e os factores extraídos explicam entre $50.9 \%$ e $67.6 \%$ da variância total. A estabilidade temporal foi moderada a muito forte $(r=$ .66 a $r=.92)$. Os doentes tem valores significativamente mais elevados na EEI e na EEE do que os controlos, fortalecendo a validade de construto. As correlações moderadas (de $r=.50$ a $r=.64$ ) entre a EE e o Inventário Depressivo de Beck indiciam uma convergência entre estes instrumentos.

A Escala de Entrapment apresenta boas características psicométricas, sendo necessário agora ampliar a sua aplicação em contextos clínicos de carácter prospectivo.

PALAVRAS-CHAVE: Entrapment, Psicopatologia evolucionária, Características psicométricas.

\footnotetext{
1 Hospital de Magalhães Lemos, E.P.E. - Porto. smicarval@gmail.com

2 CINEICC. Faculdade de Psicologia e Ciências da Educação da Universidade de Coimbra

3 Centro Hospitalar de Trás-os-Montes e Alto-Douro, E.P.E. - Vila Real

4 Universidade Lusófona - Porto
} 


\section{Introdução}

Ao longo da evolução humana ocorreram mudanças ao nível genético determinando o aparecimento de características, traços e estratégias envolvendo os domínios anatómico, fisiológico, comportamental e emocional. Mantiveram-se no património genético, transmitindo-se às gerações seguintes as que se revelaram vantajosas para a sobrevivência da espécie, quer em contextos de sobrevivência a predadores quer na regulação social entre conspecíficos. No homem, evoluindo em âmbitos sociais cada vez mais exigentes, foi particularmente benéfica a selecção de estratégias reguladoras dos relacionamentos e conflitos entre conspecíficos. Na base da regulação dos comportamentos humanos encontram-se pois estratégias filogeneticamente evoluídas e.g., fuga, luta, submissão e procura de ajuda.

Do ponto de vista evolucionário convém definir o conceito de estratégia. Desde logo refere-se a um comportamento típico de espécie. As estratégias reflectem várias hipóteses de resposta que estão geneticamente codificadas e variam de espécie para espécie dependendo de múltiplos factores sócio-ecológicos (Krebs \& Davies, 1993); correspondem a um padrão de resposta psicobiológico interno em relação a um estímulo mais ao menos específico e à sua interpretação (Gilbert, 1984, 1992). Estas estratégias são extremamente variadas e vão desde a fuga ou submissão mais imediata até elaboração de alianças.

As estratégias de submissão defensiva, que pertencem ao grupo de estratégias utilizadas entre conspecíficos, não com predadores, orientam o indivíduo a adoptar certos comportamentos que visam genericamente: a vigilância de ameaças sociais (por dominantes); reduzir ou moderar a aproximação a recursos (e.g., parceiro, alimentos ou território); evitar a escalada de conflitos até lutas com ferimentos graves ou morte.

\subsection{Conceito de entrapment}

A estratégia de fuga é um dos mecanismos mais antigos para escapar ou evitar conflitos, quer perante predadores quer entre conspecíficos. Quando as estratégias de defesa estão altamente activadas mas inibidas ou adiada a sua concretização, esse facto leva a perturbação emocional, a reacções comportamentais anómalas e respostas fisiológicas anormais. Estudos em modelos animais (MacLean, 1990) sugerem que um animal derrotado num conflito mas com possibilidade de fuga está muito mais protegido do que um animal nas mesmas condições mas sem fuga possivel. Dixon, Fish, Huber, \& Walser (1989) sugeriram que, num contexto competitivo, se o derrotado que deveria fugir ou escapar é impedido de o fazer, entra num estado de fuga peculiar, com redução da motivação e inibição psicomotora dando lugar a um tipo de comportamento defensivo 
social, a que chama fuga inibida ou adiada (arrested flight). Em várias espécies, a resposta de fuga é activada frequentemente por ataques, ou mesmo só pela presença de dominantes a que os subordinados respondem com sinalização de defesa agressiva e fuga. No estado de escape ou fuga inibida esta resposta não acontece, ocorrendo então comportamentos anómalos como supressão do comportamento exploratório (especialmente aproximação), posturas de submissão com imobilização (freezing), chegando o animal a ficar nos limites do território, separado do ambiente e imóvel.

O conceito de learned helplessness (Seligman, 1975) pode ser considerado próximo do conceito de entrapment, enquanto neste existe uma forte propensão para o escape ou fuga e vivências de derrota e humilhação, naquele isso não parece acontecer, referindo-se mais a incontrolabilidade do stress (Gilbert, 1992) com uma resposta de desistência e um sentimento de impotência, e ainda que em certo momento possa haver possibilidade de escape, o indivíduo não acredita que pode vencer.

No caso do entrapment, os sujeitos sob intenso stress, estão fortemente motivados para fuga ou escape porém, por vários motivos, não existe implementação dessa estratégia.

Define-se então entrapment como uma estratégia de submissão ou subordinação defensiva na qual a propensão para a fuga está intensamente activada mas inibida na sua execução.

Quando as estratégias evolucionárias (e.g., luta, fuga, submissão ou procura de ajuda) estão fortemente activadas pelos estímulos específicos a que respondem mas bloqueadas, ou existindo reacção esta não é suficiente para reduzir o stress, nesse caso, quer em modelos animais quer em humanos, os indivíduos tendem a cair em estados de stress crónico. Como consequência resultam anomalias na regulação do humor por mecanismos de feedback psicobiológico, seja por desregulação do eixo adreno-hipotálamo-hipofisário (Dixon \& Fish, 1998; Gilbert, 2001), por alterações neuroquímicas mediados pelo sistema límbico ou por processos fisiológicos primitivos (i.e. cortisol e serotonina) (Bale, 2006; Gilbert, 2001; Marks, 1987; Panskepp, 1998).

Foi Unger (1984) com o conceito de aprisionamento e Gilbert (1989) com o conceito de bloqueio ou inibição de fuga (blocked scape) que primeiro tentaram operacionalizar o conceito de entrapment na clínica. No contexto humano os estímulos para activação da resposta de fuga são genericamente certos acontecimentos e dificuldades de vida. Neste contexto, numa tentativa de operacionalização do conceito, o entrapment pode ser encarado como uma resposta a estímulos externos ou ambientais claros como no entrapment externo e.g., 
mulher divorciada, com 2 filhos pequenos, sem suporte social, sem outro rendimento, sujeita humilhação diária no local de trabalho do qual não pode sair. Quando a activação para a fuga está motivada por estados emocionais aversivos denomina-se entrapment interno e.g., forte motivação para sair de uma relação de violência conjugal, mas vergonha pelo que a família pode pensar. Brown, Harris \& Hepworth (1995) aplicando o conceito de entrapment na entrevista de acontecimentos e dificuldades de vida (LEDS, Life Events and Difficulties Schedule) definem as dimensões dos acontecimentos e dificuldades de vida em quatro tipos: perda, humilhação, entrapment e ameaça. Verificando que dificuldades de vida definidas na dimensão entrapment eram mais fortes preditores de depressão do que os acontecimentos que envolviam perda.

Com muita frequência, a maior parte dos problemas de vida associadas a stress crónico (Morris \& Morris, 2000) e depressão (Brown \& Harris, 1978; Brown, Bifulco \& Harris, 1987), podem ser vistos como situações de sem-saída, de inibição de resposta de fuga ou entrapment i.e., o indivíduo está altamente motivado para sair de uma dada situação (activação do mecanismo de fuga), mas sente-se incapaz de o fazer. Brown et al., (1995) exploraram esta possibilidade e verificaram que acontecimentos de vida relacionados com perda com um sentido de humilhação (e.g., em contextos de submissão ou subordinação), bem como acontecimentos de vida relacionados com sentimento de sem-saída ou entrapment foram preditores de depressão mais fortes do que os acontecimentos de vida relacionados com perda isolada. Kendler, Hettema, Butera, Gardner \& Prescott (2003) verificaram num estudo prospectivo com 7322 gémeos que altos níveis de entrapment eram preditores de episódios mistos de ansiedade e depressão.

Gilbert \& Allan (1998) durante o desenvolvimento da escala para avaliação do entrapment verificaram que elevada motivação para a fuga associado ao sentimento de sem-saída ou entrapment estava altamente correlacionado com depressão, sentimento de derrota e desesperança. Wilner \& Goldstein (2001) concluíram que a ocorrência de depressão em mães em situação de forte stress de carácter permanente (filhos com deficiências graves) era mediada por entrapment interno, entrapment externo e sentimento de derrota. Entre nós, num estudo exploratório estudou-se o efeito de variáveis evolucionárias de tipo traço, tais como submissão e comparação social e estados de entrapment em amostras da população geral e de doentes deprimidos. Nos doentes verificou-se que o entrapment externo só por si explicava $48.3 \%$ da variância do Inventário Depressivo de Beck (BDI); na população geral o entrapment interno foi o maior preditor, explicando 55.3\% da variância do BDI (Carvalho, Pinto-Gouveia, Pimentel \& Maia, 2007). Rooke \& Birchwood (1998) verificaram em doentes psicóticos que o sentimento de subordinação (por perda de estatuto social) bem como o 
entrapment relacionado com o facto de se estar a sofrer uma doença psicótica estava altamente correlacionado com depressão pós-psicose. Baumeister (1990) concluiu que o desejo de escapar de uma dada situação, associada à percepção de impossibilidade de o fazer, era frequentemente central no suicídio. Existem acerca do facto várias referências a casos individuais de doentes deprimidos que melhoram do humor quando decidem suicidar-se, acreditando que assim, podem libertar-se do seu sofrimento (Williams, 1997).

Utilizando grupos de discussão (focus groups) de pessoas deprimidas verificouse que o sentimento de entrapment, produzido quer pela própria depressão (entrapment interno), quer pelos acontecimentos de vida associados é comum no episódio depressivo (Gilbert, 2003). No modelo do grito de dor (CoP, cry of pain) para o comportamento suicida (Williams, 2001; O'Connor, 2003), este é entendido como uma resposta comportamental para uma situação stressante com três componentes: sentimento de derrota, ausência de hipótese de fuga (entrapment), ausência de possibilidade de ajuda externa (suporte social). Além disso, o modelo prevê um papel mediador para o entrapment na relação entre derrota e ideação suicida, e um papel moderador dos factores de apoio externo (suporte social) na relação entre entrapment e ideação suicida. Vários estudos têm replicado a validade do modelo em diversos contextos. O'Connor (2003) encontrou base para o modelo num estudo de caso-controlo para comportamento suicida e Rasmussen et al., (2009) verificaram igualmente a validade deste modelo numa amostra de sujeitos com um primeiro episódio, e episódios repetidos de auto-mutilação.

Estar em entrapment foi um factor de risco para tentativa de suicida entre prostitutas em Goa (Shahmanesh et al., 2009). Igualmente, no âmbito das perturbações do comportamento alimentar se verificou que o entrapment interno foi um preditor de sintomatologia depressiva (Troop \& Baker, 2008). Na mesma linha, Leahy (2000) desenvolveu a teoria da "perda irreparável" ou "custos irreparáveis" (sunk costs), sugerindo que quando as pessoas sentem que investiram muito numa situação (e.g., emprego, carreira ou relações) tendem a manter-se nela devido ao muito que perderiam se saíssem, ainda que se encontrem em entrapment i.e., possam estar a perder economicamente, em grande adversidade ou a sofrer grande stress.

O sentimento de entrapment pela própria doença ou por certos estados emocionais denomina-se entrapment interno, isto é, o estado de sentir-se sem-saída mas manter o desejo de sair de estados emocionais dolorosos está associado significativamente com depressão (Gilbert \& Allan, 1998; Gilbert \& Gilbert, 2003), depressão na esquizofrenia (Rooke e Birchwood, 1998) e é uma razão comum para o suicídio (Baumeister, 1990). 
Gilbert, Gilbert \& Irons (2004) cita um exemplo de Seligman (1975), de um prisioneiro de guerra que estava convencido que os aliados ganhariam a guerra e o libertariam dentro de alguns meses. Esta esperança, que funcionava como uma fantasia de evitamento do seu estado de prisioneiro, manteve o seu humor estabilizado. Quando se tornou claro que esse facto não iria acontecer entrou em depressão e morreu pouco depois. Verifica-se frequentemente que mesmo para pessoas gravemente deprimidas, logo que fazem um plano para se matar, e evitar desse modo o sofrimento, o seu humor pode elevar-se. Tais observações e dados de investigação sugerem complexas interacções nos mecanismos defensivos (de fuga/evitamento) que não podem inteiramente ser explicados pela teoria do controlo, ao menos por uma vez uma pessoa escolhe o suicídio e dá a si própria todo o controlo sobre os objectivos da sua vida. Igualmente, alguns investigadores sugerem a necessidade de pesquisa mais focalizada sobre o relacionamento íntimo entre o sistema límbico, mecanismos de fuga e humor (Gilbert et al., 2004).

O foco em comportamentos defensivos activados, mas demoradamente inibidos na resposta (e.g., luta e fuga) como uma fonte de stress crónico e depressão é relativamente nova, embora tenha muitas relações com a teoria do desespero ou desamparo aprendido como referido anteriormente.

Tornou-se assim imprescindivel desenvolver uma estratégia empírica de investigação para testar as predições específicas destes modelos, que passa pelo desenvolvimento de escalas e questionários específicos particularmente para avaliar o entrapment interno e externo.

A Escala de Entrapment original foi desenvolvida a partir do trabalho com doentes deprimidos e publicada por Gilbert \& Allan em 1998. De facto é composta por duas escalas no mesmo suporte, a Escala de Entrapment Interno e a Escala de Entrapment Externo. A Escala de Entrapment foi validada num grupo de estudantes $(N=299)$ e num grupo de doentes $(N=86)$, tendo sido avaliadas as suas propriedades psicométricas e a estrutura factorial. As escalas revelaram um bom indice de consistência interna, com um alfa de Cronbach nas duas escalas, nos dois géneros e nos dois grupos oscilando entre .82 e .94. Para averiguar a validade de construto os autores conduziram uma análise de componentes principais com rotação oblíqua (Oblimin), que permitiu extrair um componente na Escala de Entrapment Interno nos dois grupos. Na Escala de Entrapment Externo esse facto ocorreu na amostra de estudantes, na amostra de doentes foram extraídos dois factores com eigenvalues superiores a 1.00 e explicando respectivamente $51.8 \%$ e $11.4 \%$ da variância. No entanto os autores, devido à correlação entre os dois factores $(r=.58)$ e ao conteúdo dos itens, efectuaram uma solução forçando um factor único. Desse modo 
obtiveram um único factor com cargas factoriais nos itens iguais ou superiores a . 56 sugerindo uma solução unidimensional que passou a explicar $51.8 \%$ da variância do BDI. Relativamente à validade convergente foram obtidas correlações de .64 e .65 respectivamente entre as escalas de Entrapment Interno e de Entrapment Externo e a CES-D (Centre for Epidemiological Studies Scale) na amostra de estudantes; e de .62 e .54 entre as mesmas escalas e sintomatologia de depressão (BDI) na amostra de doentes. Desde a sua publicação, a Escala de Entrapment foi usada em vários estudos como medida do entrapment quer em amostras cínicas quer em amostras da população geral (Willner \& Goldstein, 2001; Gilbert et al., 2002; Sturman \& Mongrain, 2005; Martin, Gilbert, McEwan, \& Irons, 2006; Troop \& Baker, 2008; Rasmussen et al., 2009; Shahmanesh et al., 2009).

Tanto quanto é do nosso conhecimento não foram realizados estudos psicométricos da Escala de Entrapment na população portuguesa.

\section{Objectivos}

$\mathrm{Na}$ tentativa de ultrapassar o défice referido, o presente estudo procura em primeiro lugar traduzir e adaptar para a língua portuguesa a Escala de Entrapment. Em segundo lugar pretende investigar a estrutura factorial da versão portuguesa da Escala de Entrapment Interno e Externo num grupo de estudantes, num grupo da população geral e num grupo de doentes com depressão em relação às suas experiências de entrapment. Em último lugar, o presente estudo pretende avaliar as propriedades psicométricas da estrutura factorial obtida, especificamente a análise dos itens e consistência interna, fidelidade teste-reteste e, ainda, explorar a validade convergente deste instrumento usando medidas de depressão.

\section{Metodologia}

\subsection{Participantes}

O presente estudo é constituído por uma amostra com três grupos de sujeitos. Um grupo era formado por estudantes, outro constituído por sujeitos da população geral e outro grupo constituído por doentes com diagnóstico de episódio depressivo major seguidos em consulta externa de psiquiatria. 


\section{Grupo de Estudantes}

O grupo de estudantes era composto por 608 sujeitos (428 do género feminino e 180 do género masculino) provenientes de instituições de Ensino Superior e de escolas profissionais da região Centro e Norte do País, a idade média foi de 20.7 anos (DP $=2.9$ ) e a escolaridade média foi de 13.4 anos (DP $=1.6$ ).

\section{Grupo da População Geral}

O grupo de sujeitos da população geral era composto por 406 sujeitos (248 do género feminino e 158 do género masculino), proveniente de várias empresas privadas e instituições públicas do Centro e Norte do País, a idade média foi de 37.7 anos ( $D P=10.2$ ); sendo a escolaridade média de 12.9 anos (DP = 4.0).

\section{Grupo de Doentes}

O grupo de 106 doentes ( 78 do género feminino e 28 do género masculino) consistia em doentes com um novo episódio de depressão major que recorreram à consulta externa do Hospital de Magalhães Lemos - Porto entre Janeiro de 2006 e Dezembro de 2006. A idade média foi de 37.9 anos (DP = 10.6); sendo a escolaridade média de 10.9 anos ( $D P=4.2)$.

No grupo de estudantes foi utilizada uma amostra de conveniência colhida em instituições públicas e privadas de ensino. A amostra foi obtida entre estudantes de universidades e escolas profissionais do Norte e Centro do País. Foi pedido autorização à direcção das instituiç̃ões e após esta foram contactados professores de referência que permitissem a utilização de parte do seu tempo lectivo para a presente investigação. Os alunos foram informados e procedeuse à marcação de datas. Nesse dia, os alunos presentes foram esclarecidos dos objectivos da investigação e foram convidados a participar, aqueles que aceitaram assinaram um termo de consentimento, realçando a participação voluntária e a confidencialidade. Os questionários foram preenchidos na presença de um dos investigadores.

No grupo da população geral foi utilizada uma amostra de conveniência colhida em instituições públicas e empresas privadas. A amostra foi obtida entre trabalhadores de três empresas privadas e entre funcionários de três instituições públicas do Norte e Centro do País. Foi pedida autorização à direcção das empresas instituições e após esta, foram contactados quadros intermédios para a ligação aos trabalhadores e marcação de datas. Nesse dia, os trabalhadores foram esclarecidos dos objectivos da investigação e foram convidados a participar, aqueles que aceitaram assinaram um termo 
de consentimento, realçando a participação voluntária e a confidencialidade. Os questionários foram preenchidos no mesmo dia ou em data posterior na presença de um dos investigadores

A amostra de doentes deprimidos foi obtida na consulta externa de psiquiatria do Hospital de Magalhães Lemos. Os doentes enviados pelo médico de família com diagnóstico de depressão e que na entrevista clínica confirmaram as suspeitas, foram sujeitos a avaliação diagnóstica de depressão major com a SCID-I. Todos os doentes com o diagnóstico de episódio de depressão major e com BDI igual ou superior a 20, eram elegíveis para entrar no estudo. Aos doentes com diagnóstico de episódio depressivo major e sem critérios de exclusão (i.e., perturbação por dependência de substâncias e perturbações psicóticas) foi solicitada a sua participação na investigação e explicados os objectivos. Dos 115 doentes elegíveis, 5 não aceitaram participar e 4 faltaram na data marcada para o preenchimento do protocolo. Os doentes que aceitaram, assinaram o termo de consentimento, onde se realçava a voluntariedade da participação e confidencialidade das respostas. O presente projecto de investigação foi autorizado pela comissão de ética do hospital.

\subsection{Instrumentos}

A Structured Clinical Interview for DSM-IV Axis I Disorders, (SCID-I) (First, Spitzer, Gibbon \& Williams, 1997) Foi utilizada para estabelecer o diagnóstico. Foi usada a versão traduzida e adaptada para a língua portuguesa (Portugal) por Maia, Pinto-Gouveia, Carvalho, Pimentel \& Guimarães (2006) (in press).

O Inventário de Depressão de Beck (BDI) foi utilizado para a avaliação da sintomatologia depressiva (Beck, Steer \& Garbin, 1988). O BDI é utilizado para avaliar a intensidade da depressão ao longo do tempo sobretudo em doentes deprimidos em tratamento (Barkham et al, 2008), para distinguir indivíduos deprimidos dos não-deprimidos em contextos psiquiátricos (Medina, Echeburúa \& Aizpiri, 2007) e médicos (Rowan et al, 2005); é igualmente usado para avaliação de sintomas depressivos em populações não clínicas, em estudantes (Gorenstein, Andrade, Zanolo, \& Artes, 2005; Lim et al, 2008; O'Connor, Berry, Weiss \& Gilbert, 2002) ou na população geral (Appleton et al, 2008). O BDI é formado por 21 itens que procuram avaliar o estado depressivo nos seus aspectos afectivos, motivacionais, cognitivos e fisiológicos. O inventário utilizado é uma versão validada para a população portuguesa e mostra correlacionar-se de modo altamente significativo com o critério clínico de depressão (Vaz Serra \& Pio-Abreu, 1973a; 1973b; 1973C). Neste estudo apresentou um alfa de Cronbach de .88 na amostra de estudantes, de .89 na amostra da população geral e de .95 na amostra de doentes. 


\section{Escalas de Entrapment (EE)}

A Escala de Entrapment é de facto composta por duas escalas, a Escala de Entrapment Interno (EEI) e a Escala de Entrapment Externo (EEE), outros investigadores para além de utilizarem os valores destas duas escalas utilizam também o entrapment total, somatório de ambas (Sturman \& Mongrain, 2008; Rasmussen et al, 2009).

\section{Escala de Entrapment Interno (EEI)}

A EEI procura captar a motivação para a fuga determinada por estados emocionais aversivos. Tem 6 itens e.g., "Gostaria de me livrar dos meus pensamentos e sentimentos", tendo uma pontuação máxima possivel de 24 . Na versão original apresenta um alfa de Cronbach de .93 para a amostra de estudantes e .86 para a amostra de doentes deprimidos (Gilbert \& Allan, 1998).

\section{Escala de Entrapment Externo (EEE)}

A EEE procura abordar situações em que o desejo ou motivação para a fuga ou escape é estimulado por acontecimentos externos. É composta por 10 itens e.g., "Não encontro uma saída para a minha situação actual", tendo uma pontuação máxima possível de 40 . Na versão original apresenta um alfa de Cronbach de .88 para a amostra de estudantes e .89 para a amostra de doentes deprimidos (Gilbert \& Allan, 1998).

\subsection{Procedimento}

Em todas as análises foi utilizado o programa SPSS versão 15.0. Foram consideradas diferenças estatisticamente significativas todos os valores com probabilidade associada inferior a .05 (Howell, 2006).

Procurámos seguir a metodologia dos autores para validação das escalas, porém foram alterados alguns procedimentos e.g., utilizámos três amostras em vez de duas na validação original. Inicialmente procedeu-se a uma análise factorial com o objectivo de verificar a dimensionalidade das escalas, tendo-se utilizado uma análise de componentes principais (ACP) como estratégia de análise adequada (Stevens, 1986; Spector, 1994; Tabachnick \& Fidell, 2007), estabelecendo como limite o valor de .4 para inclusão de um item na interpretação de um factor. Foram dois os critérios para extracção de um factor: valores próprios maiores que 1 antes da rotação (Kaiser, 1960) e utilização de um scree test (Cattel, 1966). Em todas as análises foi utilizada rotação oblíquia (Oblimin; delta = o) como na validação original. A rotação varimax replicou os resultados obtidos confirmando as soluções encontradas. 
Procedeu-se de seguida à análise da consistência interna das escalas nas três amostras através do cálculo do alfa de Cronbach, considerada a melhor estimativa de fidelidade de um teste (Nunnally, 1978). A qualidade dos itens foi verificada através do cálculo da correlação do item com o total da escala excepto o próprio item (Nunnally, 1978).

Seguidamente procedeu-se à avaliação da fidelidade teste-reteste bem como da validade convergente que foram analisadas através do coeficiente de correlação paramétrico de Pearson (Nunnally, 1978). Para isso foi estudada a estabilidade temporal das escalas num subgrupo da amostra de estudantes $(n=31)$, e noutro da população geral $(n=41)$ em que foi repetido o preenchimento dos questionários 3 semanas mais tarde. A exploração da validade convergente foi analisada por meio de um estudo de correlação entre as escalas de entrapment com a medida de sintomatologia depressiva obtida com o BDI nas três amostras.

Para avaliação da validade de construto, foi executada uma análise da variância (ANOVA) de modo a verificar se os doentes apresentavam pontuações superiores nas duas escalas, em relação aos sujeitos das outras duas amostras.

\section{Resultados}

\subsection{Validade de conteúdo}

\section{Adaptação da medida à língua portuguesa}

A adaptação da Escala de Entrapment Interno (EEI) e da Escala de Entrapment Externo (EEE) envolveu a tradução, retroversão e comparação posterior com a versão original para verificação das possíveis diferenças e retradução final. Neste passo teve-se em atenção sobretudo o conteúdo dos itens. Através de discussões entre especialistas na área da investigação e com colaboração de peritos nas línguas portuguesa e inglesa, verificou-se se o conteúdo dos itens da versão resultante da tradução tinha o sentido proposto pelos originais. O painel concluiu pela manutenção do número de itens em cada escala e cada sujeito deveria responder a cada item em escala tipo Likert de 5 pontos, de o a 4 i.e., o = não tem nada a ver comigo; 1 =é um pouco parecido com o que sinto; 2 =é moderadamente parecido com o que sinto; 3 = é praticamente como me sinto; 4 = é exactamente como me sinto. Após este passo administraram-se as escalas de auto-avaliação a uma amostra de 20 estudantes do ensino superior, foi-Ihe explicado quais os objectivos solicitandoIhe que apontassem, após terem respondido às questões, dificuldades, dúvidas, 
críticas ou comentários que entendessem relevantes. Pela adequada compreensão dos itens deste procedimento não resultaram alterações nas escalas.

\section{Estatistica descritiva}

As médias e desvios padrão para todos os valores das escalas nas três amostras são apresentados na Tabela 1. Os resultados são idênticos aos obtidos em outros estudos (Allan \& Gilbert, 1995; 1997; Gilbert \& Allan, 1994; 1998; Gilbert, 2000; O'Connor et al., 2002; Sturman \& Mongrain, 2008). Para excluir um possível efeito de género, verificámos nas três amostras a existência de diferenças nas médias dos três instrumentos por meio de uma análise da variância (ANOVA). Verificou-se que no grupo da população geral e no grupo de doentes não existiam diferenças entre os valores das três escalas nos dois géneros. No grupo de estudantes existiam diferenças significativas nos valores do BDI e da $E E E$, obtendo o género feminino maiores pontuações no BDI e o género masculino, ainda que tangencialmente, maiores pontuações na $E E E$, respectivamente para o $B D I[F=7.69(D P=6.43)$ versus $M=5.13(D P=6.40) ; F=19.79, p<.001]$ e para a $\operatorname{EEE}[M=7,84(D P=8,60)$ versus $F=6,47(D P=7.19) ; F=4,01, p=.046]$.

\subsection{Dimensionalidade}

\subsubsection{Estrutura factorial das Escalas de Entrapment Externo (EEE) e Entrapment Interno (EEI)}

\section{Estrutura factorial da EEI}

$\mathrm{Na}$ análise da dimensionalidade, efectuou-se uma Análise em Componentes Principais (ACP), de acordo com a opção assumida pelos autores da escala original (Gilbert \& Allan, 1998). O estudo da solução inicial forneceu indicação para a retenção de uma componente com eigenvalues superiores a $1 \mathrm{em}$ todas as amostras, confirmado pela análise do scree plot de Cattel. Portanto para as três amostras foi obtido um só factor, explicando $67.6 \%$ da variância total na amostra de estudantes, $65.4 \%$ na amostra da população geral e $57.5 \%$ na amostra de doentes. Nas três amostras todos os itens tiveram pontuações acima de .4. Os itens e a carga dos factores estão representados na Tabela 2. Estes resultados apontam claramente para a unidimensionalidade da escala de entrapment interno.

\section{Estrutura factorial da EEE}

$\mathrm{Na}$ amostra de estudantes foi obtido um só factor explicando $57.3 \%$ da variância total da escala, obtendo todos os itens pontuações acima de 40 . 
Contudo, na amostra da população geral foram obtidos 2 factores explicando $68.4 \%$ da variância total. Todos os itens tiveram pontuações acima de .40 pelo menos num dos dois factores. O primeiro factor explicava $58.1 \%$ da variância total e incluía um grupo de 7 itens que representam o entrapment provocado por situações externas em geral (itens 1, 2, 4, 5, 6, 7 e 9). O segundo factor explicava $10.3 \%$ da variância e englobava 3 itens que reflectiam o entrapment provocado por circunstâncias interpessoais (itens 3, 8 e 10), particularmente relações de subjugação e subordinação. A correlação dos dois factores foi de .59.

$\mathrm{Na}$ amostra de doentes ocorreu a mesma solução que na amostra da população geral, tendo sido obtidos 2 factores explicando $62.2 \%$ da variância total. Todos os itens tiveram pontuações acima de .4 pelo menos num dos dois factores. O primeiro factor explicava $50.9 \%$ da variância total e incluía exactamente os mesmos itens da amostra da população geral. O segundo factor explicava 11.3\% da variância total e era composto por consequência pelos três itens referidos (itens 3, 8 e 10) na amostra da população geral. A correlação dos dois factores foi neste caso moderada, $r=.45$. Ao forçar a extracção de um só factor resultam itens com cargas superiores a .61 (.61 a .85), na amostra da população geral e superiores a .50 (.50 a .82) na amostra de doentes. Estes dados sugerem que a EEE pode ser considerada uma avaliação unidimensional nestas amostras. Os itens, os eigenvalues, a carga dos factores bem como a variância em cada amostra estão representados na Tabela 2.

\subsubsection{Análise dos itens e consistência interna}

\section{Escala de Entrapment Interno}

A análise da qualidade dos itens revela a existência de correlações item-item moderadas a elevadas em geral. Na EEI os itens apresentam correlações de .42 a .71 na amostra de estudantes; de .45 a .69 na amostra da população geral; e de .37 a .68 na amostra de doentes. Usualmente é melhor que as correlações interitem sejam baixas a moderadas (Miles \& Gilbert, 2005). É necessária cautela na avaliação destas correlações segundo estes autores, se forem tendencialmente baixas ou negativas significa que existe uma falta de coerência na escala. Por outro lado se forem tendencialmente elevadas pode indicar redundância nos itens i.e., se um item se correlaciona tanto assim com outro, possivelmente não é necessário.

O alfa de Cronbach para a EEI no grupo de estudantes foi de .9o (.89 para o género feminino e .91 para o género masculino), para o grupo da população geral foi de .89 (.89 para o género feminino e .89 para o género masculino), e para o grupo 
de doentes foi de .81 (.79 para o género feminino e .85 para o género masculino). Estes resultados sugerem que a EEI tem excelentes valores de consistência interna para as três populações e ambos os géneros (Tabela 3). Ao analisar-mos as correlações item-total da EEl verificamos que apresentam valores elevados ( $r=.53$ a $r$ = .81) o que nos indica não ser necessário excluir nenhum item (Nunnally, 1978). A leitura do indicador de alfa de Cronbach, caso o item seja retirado revela que a exclusão de qualquer item não incrementa o valor do indicador de consistência interna (Tabela 4).

\section{Escala de Entrapment Externo}

A EEE apresenta correlações item-item entre .32 e .69; entre .30 e .74 e entre .30 e .69, respectivamente na amostra de estudantes, população geral e doentes. Tal como na EEI, a EEE apresenta correlações inter-item de moderadas a elevadas (Cohen, 1988), sugerindo valores adequados de consistência interna.

O alfa de Cronbach para a EEE na amostra de estudantes foi de .92 (.91 para o género feminino e .93 para o género masculino); para a amostra da população geral foi de .92 (.92 para o género feminino e .91 para o género masculino); e para a amostra de doentes foi de .91 (.91 para o género feminino e .91 para o género masculino). Estes resultados sugerem que a EEE tem valores altos de consistência interna para as três populações e ambos os géneros (Tabela 3). Ao analisar-mos as correlações item-total da EEE, verificamos que como regra tem valores elevados i.e., $r$ de Pearson (2-tailed) $\geq .50$ (Cohen, 1988), à excepção de dois itens que apresentam correlações moderadas: o item $3(r=.43)$ e o item 8 ( $r=$ .45) na amostra de doentes, estes dados indicam-nos não ser necessário excluir nenhum item (Nunnally, 1978). A leitura do indicador de alfa de Cronbach, caso o item seja retirado, revela que a exclusão de qualquer item não incrementa o valor do indicador de consistência interna (Tabela 5).

\subsubsection{Fidelidade teste-reteste}

Ao nivel da consistência interna, a EEI apresentou um valor de alfa de Cronbach para o reteste de .93 e .96 respectivamente, para a amostra de estudantes e da população geral; do mesmo modo a EEE apresentou valores de .95 e .96.

Num subgrupo da amostra de estudantes $(n=31)$ e noutro da população geral $(n=41)$ foi repetido o preenchimento dos questionários 3 semanas mais tarde. 0 resultado do estudo da estabilidade temporal das escalas demonstrou elevados coeficientes de correlação de Pearson ( $r=.66$ a $r=.92)$ nas duas escalas e nas duas amostras (Tabela 6). 


\subsubsection{Validade convergente}

Para avaliar a validade externa ou de critério da EEI e da EEE nas três amostras foi utilizado o BDI. Procedeu-se a um estudo de correlação entre as escalas de entrapment e o BDI nas três amostras, obtendo-se correlações iguais ou superiores a .50 ( $r=.50$ a $r=.63)$, revelando que existe uma correlação elevada e positiva (Cohen, 1988) entre ambas as escalas de entrapment e os valores do BDI nas três amostras. Esse facto aponta para a existência de um convergência, mais do que uma divergência entre as escalas de entrapment e BDI (Tabela 7).

\subsubsection{Validade de construto}

A validade de construto é a validade nobre de qualquer teste. É ela que garante que o teste mede o que se propõe medir. Quer a distribuição das médias (Tabela 1) quer a validade convergente referida anteriormente já forneciam pistas; para validar a EEl e a EEE inspeccionámos a sua distribuição nas três amostras, sendo assim, as pontuações deveriam ser superiores na amostra de doentes em relação às amostras de estudantes e da população geral. Foi realizada uma análise da variância (ANOVA) no sentido de verificar se havia diferenças nas médias da EEI e EEE estatisticamente significativas nas três amostras. Caso houvesse, deveria avaliar-se como se apresentavam essas diferenças entre os grupos, pelo que realizámos um teste de Tukey para localização das diferenças. Verificou-se que, quanto à EEI havia diferenças significativas entre os três grupos (Tabela 8). Os doentes tinham valores mais elevados do que os estudantes e que a população geral. Por outro lado, os estudantes tinham pontuações significativamente mais elevadas do que a população geral (Tabela 8).

Quanto à EEE constatou-se que havia diferenças significativas entre os doentes e os outros dois grupos (Tabela 8). Os doentes tinham valores mais elevados do que os estudantes e do que a população geral. Neste caso, nenhum dos outros grupos apresentou diferenças estatisticamente significativas na comparação das médias da EEE entre si (Tabela 8).

\section{Discussão}

Esta investigação procurou contribuir para a validação da versão portuguesa da Escala Entrapment Interno (EEI) e da Escala de Entrapment Externo (EEE) bem como para o estudo das suas características psicométricas, especificamente da estrutura factorial, consistência interna, estabilidade temporal, validade convergente e de construto em três amostras, uma de estudantes, uma da população geral e uma de doentes com depressão. 
Quanto à validade de construto da EEl e da EEE, foram utilizados procedimentos estatísticos que permitissem compreender a organização dos itens que compõem a medida, através de um método estatístico multivariado de cariz exploratório. Este cálculo revelou a existência de uma estrutura unidimensional subjacente aos 6 itens da EEI e aos 10 itens que compõem a EEE.

Quanto à ocorrência de dois factores na EEE na amostra da população geral e dos doentes, é de realçar em particular a natureza dos itens: "Estou envolvido(a) numa relação da qual não posso sair" (item 3) "Gostaria de me ver livre de certas pessoas poderosas e dominadores presentes na minha vida" (item 8) "Sinto-me subjugado(a) por outras pessoas" (Item 10). Ao considerar-se a natureza do entrapment é interessante verificar que existe uma separação entre a percepção de entrapment provocado por situações e relacionamentos pessoais. Os motivos pelos quais as pessoas se sentem em entrapment pode ser relevante, por exemplo estar em entrapment por falta de recursos pode ter um efeito diferente de estar em entrapment por relacionamentos caracterizados por subjugação. Estes dados poderiam levar a investigar futuramente o impacto de diferentes formas de entrapment. Apesar destes dados parece prematuro caracterizar estes factores como duas subescalas diferentes nestas amostras. A correlação entre os dois factores obtidos nas duas amostras, $r=.59$ e $r=.45$ respectivamente da população geral e doentes, é suficientemente forte para considerar que é possivel substituir os dois por um só factor (Nunnally \& Bernstein, 1994).

No presente estudo, foi encontrado um alfa de Cronbach elevado para o total da EEI e da EEE, indicador de excelente consistência interna das escalas. Este resultado está de acordo com o que foi reportado no estudo original da escala por Gilbert \& Allan (1998), que encontraram indicadores de fidelidade elevados para o total das escalas.

Na nossa amostra, a EEI e a EEE apresentaram bons índices de fidelidade testereteste, mostrando uma boa estabilidade temporal para um intervalo de três semanas.

Em relação à validade convergente, na nossa investigação foi encontrado um coeficiente de correlação elevado e estatisticamente significativo com o BDI como medida da sintomatologia depressiva nas três amostras. Este resultado aponta pois para uma boa validade convergente da EEI e da EEE e suporta os dados encontrados noutros estudos, nos quais a EEI e a EEE aparecem positivamente associada a instrumentos que avaliam a sintomatologia depressiva e outra psicopatologia para além do BDI e.g., CES-D, Hopelessness Scale, Depressive Experiences Questionnaire (DEO), Fawcett-Clark Pleasure Capacity Scale (FCPCS), Mood and Anxiety Symptoms Questionnaire (MASO) ou a SHAPS, Snaith-Hamilton Pleasure Scale). 
O conceito de entrapment não é novo (Baumeister, 1990) e têm sido estudado em modelos animais e humanos de psicopatologia há mais de duas décadas (Price, 1972; Henry, 1982; Gilbert, 1989, 1992; Dixon et al., 1989). Perante acontecimentos e dificuldades de vida, o indivíduo reage naturalmente (e.g., escapando, evitando, lutando ou procurando ajuda) de modo a superar a situação, esse é o comportamento esperado pelos sujeitos saudáveis. Outra alternativa é a "não-reacção", o sujeito em grande stress e profundamente motivado para a fuga ou escape, adia a acção de fuga, nesse caso o sujeito está em entrapment e tem altas probabilidades de adoecer (Brown et al., 1995; Gilbert et al., 2004).

\section{Limitações e investigações futuras}

Os nossos resultados devem ser apreciados tendo em conta algumas limitações metodológicas. Em primeiro tratou-se de um estudo transversal, pelo que a avaliação do dinamismo das escalas em apreço não pôde ser efectuado. Em segundo lugar, a dimensão da amostra de doentes esteve no limite do aceitável para estudos de análise factorial. Por último, utilizámos metodologia pouco ampla para avaliação da validade convergente, pensamos que no futuro se deverá alargar a avaliação a outras medidas de vulnerabilidade emocional e stress; por outro lado, parece-nos necessário ampliar a aplicação da EEI e da EEE em contextos clínicos particularmente em estudos prospectivos.

\section{Conclusões}

A Escala de Entrapment (EEI e EEE) permite a avaliação futura do entrapment, sendo o primeiro instrumento a utilizar a autoavaliação do impacto emocional das situações activadoras da resposta de fuga ou escape. As escalas apresentam sinais de alta consistência interna, parecendo ambas favorecer fortemente uma estrutura unidimensional. A versão portuguesa das Escalas de Entrapment mostrou-se associada a sintomatologia depressiva; globalmente, as características psicométricas obtidas neste estudo, replicam os resultados obtidos pelos seus autores em amostras britânicas, demonstrando as escalas ter adequadas características psicométricas para serem utilizadas na população portuguesa quer em contextos clínicos quer não clínicos. 


\section{Bibliografia}

Allan, S., \& Gilbert, P. (1995). A Social Comparison Scale: psychometric properties and relationship to psychopathology. Personality Individual Differences, 19, 293-299.

Allan, S., \& Gilbert, P. (1997). Submissive behaviour and psychopathology. British Journal of Clinical Psychology, 36, 467-488.

Appleton, K. M., Gunnell, D., Peters, T. J., Ness, A. R., Kessler, D., \& Rogers, P. J. (2008). No clear evidence of an association between plasma concentrations of $n-3$ long-chain polyunsaturated fatty acids and depressed mood in a non-clinical population. Prostaglandins Leukot Essent Fatty Acids, 78, 337-42.

Bale T. L. (2006) Stress sensitivity and the development of affective disorders. Behavioral Neuroendocrinology, 50, 529-533.

Barkham, M., Stiles, W. B., Connell, J., Twigg, E., Leach, C., Lucock, M., Mellor-Clark, J., Bower, P., King, M., Shapiro, D. A., Hardy, G. E., Greenberg, L., \& Angus, L. (2008). Effects of psychological therapies in randomized trials and practice-based studies. British Journal of Clinical Psychology, 47, 397-415.

Baumeister, R. F. (1990). Suicide as scape from self. Psychological Review, 97, 90-133.

Beck, A. T., Steer, R. A., \& Garbin, M. G. (1988). Psychometric properties of the Beck Depression Inventory: twenty-five years of evaluation. Clinical Psychology Review, 8, 77-100.

Brown, G. W., \& Harris, T. O. (1978). Social origins of depression. London: Tavistock.

Brown, G. W., Bifulco, A., Harris, T. O. (1987). Life events, vulnerability and onset of depression: Some refinements. British Journal of Psychiatry, 150, 30-42.

Brown, G. W., Harris, T. O., Hepworth, C. (1995). Loss, humiliation and entrapment among women developing depression: a patient and non-patient comparison. Psychological Medicine, 25, 7-21.

Carvalho, S., Pinto-Gouveia, J., Pimentel, P., \& Maia, D. (2007). Mediation of depression by entrapment perception: through an evolutionary exploration (Book of Abstracts). World Congress of Behavioural and Cognitive Therapies, Barcelona.

Cattel, R., B. (1966). The scree test for the number of factors. Multivariate Behavioral Research, $22,44-50$

Cohen, J. (1988). Statistical power analysis for the behavioral sciences (2nd ed.). Hillsdale NJ: Erlbaum.

Dixon, A., \& Fish, H. (1998). Animal models and ethological strategies for early drug testing in humans. Neuroscience and Biobehavioral Reviews, 23, 345-358.

Dixon, A., Fish, H., Huber, C., Walser, A. (1989). Ethological studies in animals and man, their use in psychiatry. Pharmacopsychiatry, 22, 44-50.

First, M. B., Spitzer, R. L., Gibbon M., \& Williams, J. B. W. Structured Clinical Interview for DSMIV Axis I Disorders, Clinician Version (SCID-CV) (1996). Washington, D.C.: American Psychiatric Press, Inc. [Portuguese - Portugal version: Maia, A., Pinto-Gouveia, J., \& Carvalho, S. (2007)].

Gilbert, P. (1984). Depression: From Psychology to Brain State. London: Lawrence Erlbaum Associates.

Gilbert, P. (1989). Human Nature and Suffering. Hove: Lawrence Erlbaum.

Gilbert, P. (1992). Depression: The Evolution of Powerlessness. Hove: Lawrence Erlbaum and New York: Guilford Press.

Gilbert, P. (2000). The relationship of shame, social anxiety and depression: The role of the evaluation of social rank. Clinical Psychology and Psychotherapy, 7, 174-189. 
Gilbert, P. (2001). Depression and stress: A biopsychosocial exploration of evolved functions and mechanisms Stress: The International Journal of the Biology of Stress, 4, 121-135.

Gilbert, P., \& Allan, S. (1994). Assertiveness, submissive behaviour and social comparison. British Journal of Clinical Psychology, 33, 295-306.

Gilbert, P., Allan, S. (1998). The role of defeat and entrapment (arrested flight) in depression: an exploration of an evolutionary view. Psychological Medicine, 28, 584-597.

Gilbert, P., Allan, S., Brough, S., Melley, S., \& Miles, J. N. (2002). Relationship of anhedonia and anxiety to social rank, defeat and entrapment. Journal of Affective Disorders, 71, 141-51.

Gilbert, P., Gilbert, J. (2003). Entrapment and arrested fight and flight in depression: An exploration using focus groups. Psychology and Psychotherapy: Theory Research and Practice, 76, 173-188.

Gilbert, P., Gilbert, J., Irons, C. (2004). Life events, entrapments and arrested anger in depression. Journal of Affective Disorders, 79, 149-160.

Gorenstein, C., Andrade, L., Zanolo, E., Artes, R. (2005). Expression of depressive symptoms in a nonclinical Brazilian adolescent sample. Canadian Journal of Psychiatry, 50, 129-36.

Henry, J. (1982). The relation of social to biological process in disease. Social Science Medicine, 16, 369-390.

Howell, D. (2006). Statistical methods for psychology (6th ed.). USA: Thomson Wadsworth.

Kaiser, H. F. (1960). The application of electronic computers to factor analysis. Educational and Psychological Measurement, 20, 141-151.

Kendler, K. S., Hettema, J. M., Butera, F., Gardner, C. O., Prescott, C. A. (2003). Life Event Dimensions of Loss, Humiliation, Entrapment, and Danger in the Prediction of Onsets of Major Depression and Generalized Anxiety. Archives of General Psychiatry, 60, 789-796.

Krebs, J. R., Davies, N. B. (1993). An Introduction to Behavioural Ecology. Blackwell Scientific Publications.

Leahy, R. (2000). Sunk costs and resistance to change. Journal of Cognitive Psychotherapy, 14, 355-371.

Lim, J. S., Kim, S. J., Jeon, W. T., Cha, K. R., Park, J. H., \& Kim, C. H. (2008). Reliability and validity of the Korean version of Obsessive-Compulsive Inventory-Revised in a non-clinical sample. Yonsei Medical Journal, 49, 909-16.

MacLean, P. D. (1990). The triune brain in evolution: role in paleocerebral functions. New York: Plenum Press.

Marks, I. (1987). Fears, Phobias and Rituals. London: Oxford University Press.

Martin, Y., Gilbert, P., McEwan, K., Irons, C. (2006). The relation of entrapment, shame and guilt to depression, in carers of people with dementia. Aging and Mental Health, 10, 101-6.

Medina, B. R., Echeburúa, E., Aizpiri, J. (2007). Psychopathological symptoms and personality traits in alcohol-dependent patients: a comparative study. Adicciones, 19, 373-81.

Miles, J., Gilbert, P. (2005). A Handbook of Research Methods in Clinical \& Health Psychology. London: Oxford University Press.

Morriss, R., \& Morriss, E. (2000). Contextual evaluation of social adversity in the management of depressive disorder. Advances in Psychiatric Treatment, 6, 423-431.

Nunnally, J. (1978). Psychometric theory (2nd ed.). USA: McGraw Hill.

Nunnally, J., \& Bernstein, I. H. (1994). Psychometric theory (3rd ed.). New York: McGraw-Hill.

O'Connor, L. E., Berry, J. W., Weiss, J., Gilbert, P. (2002). Guilt, submission and empathy in depression. Journal of Affective Disorders, 71, 19-27.

O'Connor, R. C. (2003). Suicidal Behavior as a Cry of Pain: Test of a Psychological Model. Archives of Suicide Research, 7, 297-308. 
Panskepp, J. (1998). Affective Neuroscience. London: Oxford University Press.

Price, J. S. (1972). Genetic and phylogenetic aspects of mood variation. International Journal of Mental Health, 1, 124-144.

Price, J. S., Sloman, L., Gardner, R., Gilbert, P., \& Rohde, P. (1994). The social competition hypothesis of depression. British Journal of Psychiatry, 164, 309-135.

Rasmussen, S. A., Fraser, L., Gotz, M., Machale, S., Mackie, R., Masterton, G., McConachie, S., O'Connor, R. C. (2009). Elaborating the cry of pain model of suicidality: Testing a psychological model in a sample of first-time and repeat self-harm patients. British Journal of Clinical Psychology, 19, 15-30.

Rooke, O., Birchwood, M. (1998). Loss, humiliation and entrapment as appraisals of schizophrenic illness: a prospective study of depressed and non-depressed patients. British Journal of Psychology, 37, 259-268.

Rowan, P. J., Al-Jurdi, R., Tavakoli-Tabasi, S., Kunik, M. E., Satrom, S. L., El-Serag, H. B. (2005). Physical and psychosocial contributors to quality of life in veterans with hepatitis C not on antiviral therapy. Journal of Clinical Gastroenterology, 39, 731-6.

Seligman, M. E. P. (1975). Helplessness: On Depression, Development, and Death. San Francisco: W.H. Freeman.

Shahmanesh, M., Wayal, S., Cowan, F., Mabey, D., Copas, A., Patel, V. (2009). Suicidal behavior among female sex workers in Goa, India: the silent epidemic. American Journal of Public Health, 99, 1239-46.

Spector, P., E. (1994). Summated rating scale construction: an introduction. In Basic Measurement. Ed Lewis-Beck, M., S., pp 229-300. Sage: London.

Stevens, J. (1986). Applied multivariate statistics for the social sciences. New Jersey: Hillsdale.

Sturman, E. D., \& Mongrain, M. (2005). Self-criticism and major depression: an evolutionary perspective. British Journal of Clinical Psychology, 44, 505-19.

Sturman, E. D., \& Mongrain, M. (2008). Entrapment and perceived status in graduate students experiencing a recurrence of major depression. Canadian Journal of Behavioural Science, 40, 185-188.

Tabachnick, B., \& Fidell, L. (2007). Using Multivariate Statistics. New York: Pearson Education.

Troop, N. A., \& Baker, A. H. (2008). The specificity of social rank in eating disorder versus depressive symptoms. Eating Disorders, 16, 331-41.

Unger, R. M. (1984). Passion: An essay on personality. New York: The Free Press.

Vaz-Serra, A. S., \& Pio-Abreu, J. L. (1973a). Aferição dos quadros clínicos depressivos. I-Ensaio de aplicação do "Inventário Depressivo de Beck" a uma amostra portuguesa de doentes deprimidos. Coimbra Médica, 20, 623-644.

Vaz-Serra, A. S., \& Pio-Abreu, J. L. (1973b). Aferição dos quadros clínicos depressivos. II-Estudo preliminar de novos agrupamentos sintomatológicos para complemento do "Inventário Depressivo de Beck". Coimbra Médica, 20, 713-736.

Vaz-Serra, A. S., \& Pio-Abreu, J. L. (1973c). Análise factorial das depressões: emergência de factores numa amostra portuguesa de doentes deprimidos. Coimbra Médica, 20, 875-892.

Williams, M. (1997). Cry of Pain. London: Penguin Books

Williams, M. (2001). Suicide and attempted suicide: understanding the cry of pain (2nd ed.). London: Penguin Books.

Wilner, P., \& Goldstein, R. (2001). Mediation of depression by perceptions of defeat and entrapment in high-stress mothers. British Journal of Medical Psychology, 74, 473-485. 


\section{TABELAS}

Tabela 1 - Médias e desvios padrão para os três instrumentos nas três amostras.

\begin{tabular}{lcccccc}
\hline & \multicolumn{2}{c}{$\begin{array}{c}\text { Estudantes } \\
(\mathrm{N}=608)\end{array}$} & $\begin{array}{c}\text { População geral } \\
(\mathrm{N}=406)\end{array}$ & \multicolumn{2}{c}{$\begin{array}{c}\text { Doentes } \\
(\mathrm{N}=106)\end{array}$} \\
\cline { 2 - 7 } & $\mathrm{M}$ & $\mathrm{DP}$ & $\mathrm{M}$ & $\mathrm{DP}$ & $\mathrm{M}$ & $\mathrm{DP}$ \\
\hline Entrapment Interno & 4.59 & 5.27 & 3.34 & 4.54 & 14.02 & 6.88 \\
Entrapment Externo & 5.89 & 7.73 & 5.97 & 7.59 & 22.06 & 10.78 \\
BDI & 7.03 & 6.95 & 6.42 & 6.75 & 26.22 & 10.12 \\
\hline
\end{tabular}

Nota: $\mathrm{BDI}=$ Inventário de Depressão de Beck 
Tabela 2 - Carga factorial dos itens das Escalas de Entrapment Interno e Externo nas três amostras.

\begin{tabular}{|c|c|c|c|c|}
\hline & & $\begin{array}{l}\text { Estudantes } \\
(\mathrm{N}=608)\end{array}$ & $\begin{array}{l}\text { População } \\
\text { geral } \\
(\mathrm{N}=406)\end{array}$ & $\begin{array}{l}\text { Doentes } \\
(\mathrm{N}=106)\end{array}$ \\
\hline \multicolumn{5}{|c|}{$\begin{array}{c}\begin{array}{c}\text { Escala de Entrapment Interno } \\
\text { Item }\end{array}\end{array}$} \\
\hline 1 & Quero libertar-me da minha forma de ser & .81 & .80 & .79 \\
\hline 2 & $\begin{array}{l}\text { Sinto-me impotente para mudar a forma } \\
\text { como sou }\end{array}$ & .82 & .83 & .76 \\
\hline 3 & $\begin{array}{l}\text { Gostaria de me livrar dos meus pensamentos } \\
\text { e sentimentos }\end{array}$ & .83 & .83 & .66 \\
\hline 4 & Sinto-me prisioneiro(a) de mim mesmo(a) & .87 & .86 & .75 \\
\hline 5 & $\begin{array}{l}\text { Gostaria de me libertar da minha forma de ser } \\
\text { e começar tudo de novo }\end{array}$ & .87 & .80 & .82 \\
\hline 6 & $\begin{array}{l}\text { Sinto que estou num buraco fundo do qual } \\
\text { não posso sair }\end{array}$ & .73 & .72 & .75 \\
\hline Eige & value & 4.06 & 3.92 & 3.45 \\
\hline Vari & ncia (\%) & 67.6 & 65.4 & 57.5 \\
\hline \multicolumn{5}{|c|}{$\begin{array}{l}\text { Escala de Entrapment Externo } \\
\text { Item }\end{array}$} \\
\hline 1 & $\begin{array}{l}\text { Estou numa situação em que sinto não ter } \\
\text { saída }\end{array}$ & .71 & .77 & .77 \\
\hline 2 & $\begin{array}{l}\text { Tenho um forte desejo de fugir de certas } \\
\text { coisas na minha vida }\end{array}$ & .76 & .84 & .73 \\
\hline 3 & $\begin{array}{l}\text { Estou envolvido(a) numa relação da qual não } \\
\text { posso sair }\end{array}$ & .61 & .61 & .50 \\
\hline 4 & $\begin{array}{l}\text { Tenho frequentemente o sentimento que deve- } \\
\text { ria simplesmente fugir para longe }\end{array}$ & .81 & .81 & .73 \\
\hline 5 & Sinto-me impotente para mudar as coisas & .80 & .82 & .80 \\
\hline 6 & Sinto-me prisioneiro(a) das minhas obrigações & .80 & .75 & .67 \\
\hline 7 & $\begin{array}{l}\text { Não encontro uma saída para a minha situ- } \\
\text { ação actual }\end{array}$ & .76 & .83 & .75 \\
\hline 8 & $\begin{array}{l}\text { Gostaria de me ver livre de certas pessoas } \\
\text { poderosas e dominadores presentes na minha } \\
\text { vida }\end{array}$ & .85 & .63 & .52 \\
\hline 9 & $\begin{array}{l}\text { Sinto um forte desejo de me ir embora e ficar } \\
\text { longe da vida que levo }\end{array}$ & .67 & .85 & .82 \\
\hline 10 & Sinto-me subjugado(a) por outras pessoas & .75 & .68 & .76 \\
\hline \multirow{2}{*}{\multicolumn{2}{|c|}{$\begin{array}{l}\text { Eigenvalue } \\
\text { Variância (\%) }\end{array}$}} & 5.73 & 5.81 & 5.09 \\
\hline & & 57.3 & 58.1 & 50.9 \\
\hline
\end{tabular}


Tabela 3 - Estatística descritiva e de fidelidade da EEI e EEE nas três amostras.

\begin{tabular}{|c|c|c|c|c|c|c|c|c|c|}
\hline & \multicolumn{3}{|c|}{$\begin{array}{c}\text { Estudantes } \\
(\mathrm{N}=608)\end{array}$} & \multicolumn{3}{|c|}{$\begin{array}{l}\text { População geral } \\
\qquad(N=406)\end{array}$} & \multicolumn{3}{|c|}{$\begin{array}{l}\text { Doentes } \\
(\mathrm{N}=106)\end{array}$} \\
\hline & $M$ & $\mathrm{DP}$ & $\ddagger$ & $M$ & DP & $\ddagger$ & $M$ & DP & $\ddagger$ \\
\hline EEI & 4.59 & 5.27 & .90 & 3.34 & 4.54 & .89 & 14.02 & 6.88 & .85 \\
\hline EEE & 5.89 & 7.73 & .92 & 5.97 & 7.59 & .92 & 22.06 & 10.78 & .89 \\
\hline
\end{tabular}

${ }^{\ddagger} \alpha$ de Cronbach

$\mathrm{EEI}=$ Escala de Entrapment Interno; $\mathrm{EEE}=$ Escala de Entrapment Externo

Tabela 4 - Valores médios, desvio padrão, correlação item-total corrigida e alfa de Cronbach se o item for excluído da Escala de Entrapment Interno para as três amostras.

\begin{tabular}{lcccc}
\hline & M & DP & ritem-total & $\alpha$ Cronbach \\
\hline Estudantes (N=608) & & & & \\
\hline El1 & .89 & 1.08 & .72 & .89 \\
El2 & .85 & 1.06 & .73 & .89 \\
El3 & .96 & 1.16 & .74 & .89 \\
El4 & .65 & 1.05 & .79 & .88 \\
El5 & .86 & 1.21 & .81 & .87 \\
El6 & .37 & .81 & .63 & .90 \\
\hline População Geral (N=406) & & & & \\
\hline El1 & .65 & .97 & .71 & .87 \\
El2 & .61 & .96 & .74 & .87 \\
El3 & .70 & .98 & .74 & .87 \\
El4 & .47 & .89 & .78 & .87 \\
El5 & .62 & 1.02 & .71 & .88 \\
El6 & .30 & .80 & .61 & .89 \\
\hline Doentes (N=106) & & & & \\
\hline El1 & & & .67 & .82 \\
El2 & 2.03 & 1.50 & .67 & .83 \\
El3 & 2.24 & 1.46 & .64 & .85 \\
El4 & 2.64 & 1.40 & .53 & .83 \\
El5 & 2.24 & 1.60 & .63 & .81 \\
El6 & 2.55 & 1.60 & .71 & .83 \\
\hline
\end{tabular}


Tabela 5- Valores médios, desvio padrão, correlação item-total corrigida e alfa de Cronbach se o item for excluído da Escala de Entrapment Externo para as três amostras.

$\underline{408}$

\begin{tabular}{|c|c|c|c|c|}
\hline & $M$ & DP & $r$ item-total & $\alpha$ Cronbach \\
\hline \multicolumn{5}{|c|}{ Estudantes $(\mathrm{N}=608)$} \\
\hline EE1 & .35 & .81 & .64 & .91 \\
\hline $\mathrm{EE} 2$ & 1.10 & 1.17 & .70 & .91 \\
\hline $\mathrm{EE}_{3}$ & .39 & .87 & .53 & .91 \\
\hline $\mathrm{EE}_{4}$ & .88 & 1.17 & .75 & .90 \\
\hline EE5 & .77 & 1.04 & .74 & .90 \\
\hline EE6 & .77 & 1.03 & .69 & .91 \\
\hline $\mathrm{EE} 7$ & .55 & .96 & .79 & .90 \\
\hline EE8 & .77 & 1.14 & .60 & .91 \\
\hline EE9 & .75 & 1.14 & .76 & .90 \\
\hline EE10 & .55 & .90 & 69 & .91 \\
\hline \multicolumn{5}{|c|}{ População Geral $(\mathrm{N}=406)$} \\
\hline EE1 & .33 & .78 & .70 & .91 \\
\hline EE2 & .83 & 1.09 & .79 & .90 \\
\hline EE3 & .29 & .73 & .54 & .92 \\
\hline $\mathrm{EE}_{4}$ & .69 & 1.11 & .75 & .91 \\
\hline $\mathrm{EE}_{5}$ & .68 & 1.03 & .77 & .90 \\
\hline EE6 & .83 & 1.09 & .68 & .91 \\
\hline $\mathrm{EE} 7$ & .53 & 1.02 & .76 & .90 \\
\hline EE8 & .72 & 1.14 & .56 & .92 \\
\hline EE9 & .64 & 1.11 & .80 & .90 \\
\hline EE10 & .42 & .85 & .62 & .91 \\
\hline \multicolumn{5}{|c|}{ Doentes $(\mathrm{N}=106)$} \\
\hline EE1 & 2.10 & 1.41 & .69 & .87 \\
\hline EE2 & 2.75 & 1.44 & .63 & .87 \\
\hline $\mathrm{EE}_{3}$ & 1.08 & 1.56 & .43 & .89 \\
\hline $\mathrm{EE}_{4}$ & 2.58 & 1.59 & .63 & .87 \\
\hline $\mathrm{EE}_{5}$ & 2.55 & 1.38 & .72 & .87 \\
\hline EE6 & 2.49 & 1.49 & .57 & .88 \\
\hline $\mathrm{EE} 7$ & 2.58 & 1.45 & .67 & .87 \\
\hline EE8 & 1.77 & 1.76 & .45 & .89 \\
\hline EE9 & 2.34 & 1.59 & .75 & .86 \\
\hline EE10 & 1.79 & 1.66 & .71 & .87 \\
\hline
\end{tabular}


Tabela 6 - Teste-reteste, coeficientes de correlação $r$ de Pearson, nas duas escalas (EEI e EEE) na amostra de estudantes e da população geral.

\begin{tabular}{lcc} 
& Estudantes & População geral \\
\cline { 2 - 3 } & $r$ & $r$ \\
\hline Entrapment Interno & $.80^{*}$ & $.92^{*}$ \\
Entrapment Externo & $.66^{*}$ & $.81^{*}$ \\
\hline
\end{tabular}

Nota: ${ }^{*}=p<.01$

Tabela 7 - Coeficientes de correlação $r$ de Pearson entre as duas escalas (EEI e EEE) e o BDI nas três amostras.

\begin{tabular}{lccc}
\hline & $\begin{array}{c}\text { Estudantes } \\
(\mathrm{N}=608)\end{array}$ & $\begin{array}{c}\text { População Geral } \\
(\mathrm{N}=406)\end{array}$ & $\begin{array}{c}\text { Doentes } \\
(\mathrm{N}=106)\end{array}$ \\
\cline { 2 - 4 } & $r$ & $r$ & $r$ \\
\hline Entrapment Interno & $.51^{* *}$ & $.63^{* *}$ & $.53^{* *}$ \\
Entrapment Externo & $.50^{* *}$ & $.64^{* *}$ & $.64^{* *}$ \\
\hline
\end{tabular}

Nota: ${ }^{* *}=p<.001 ; \mathrm{BDI}=$ Inventário de Depressão de Beck

Tabela 8- Médias, desvios padrão, testes $F$ univariados e comparação entre grupos para as três amostras para a EEI e EEE.

\begin{tabular}{|c|c|c|c|c|c|c|c|c|c|}
\hline & \multicolumn{2}{|c|}{$\begin{array}{l}\text { Estudantes } \\
(\mathrm{N}=608)\end{array}$} & \multicolumn{2}{|c|}{$\begin{array}{l}\text { População geral } \\
\qquad(\mathrm{N}=406)\end{array}$} & \multicolumn{2}{|c|}{$\begin{array}{l}\text { Doentes } \\
(\mathrm{N}=106)\end{array}$} & \multirow[t]{2}{*}{$F$} & \multirow[t]{2}{*}{$p$} & \multirow[t]{2}{*}{ Post-hoc } \\
\hline & $M$ & DP & $M$ & DP & $M$ & $\mathrm{DP}$ & & & \\
\hline $\mathrm{EEI}$ & 4.59 & 5.27 & 3.34 & 4.54 & 14.02 & 6.88 & 182.30 & .000 & $\mathrm{D}>\mathrm{PG}^{* *} ; \mathrm{D}>\mathrm{E}^{* *}>\mathrm{PG}^{*}$ \\
\hline EEE & 6.89 & 7.74 & 5.97 & 7.59 & 22.06 & 10.78 & 181.56 & .000 & $\mathrm{D}>\mathrm{PG}^{* *} ; \mathrm{D}>\mathrm{E}^{* *}>\mathrm{PG}^{n s}$ \\
\hline
\end{tabular}

Nota: ${ }^{* *} \mathrm{p}<.001 ;{ }^{*} \mathrm{p}<.01 ;{ }^{n s}=$ não significativo 


\section{Entrapment - concept, definition and psychometric characteristics of Portuguese version for the Entrapment Scale}

The role of evolutionary strategies in psychopathology has been widely studied in recent years, particularly the mechanisms of fight and flight.

Entrapment means the inhibition of escape strategy when it's psychobiologically activated. Life contexts with characteristics of entrapment are linked to stress, anxiety, depression and suicide. This work presents the psychometric characteristics of the Entrapment Scale (ES) originally developed by Gilbert \& Allan (1998). It consists of two scales: the Internal Entrapment Scale (IES) related to activation to escape, motivated by aversive emotional states and External Entrapment Scale (EES), when the desire to escape is stimulated by external events. The scale was evaluated in three samples: students $(N=608)$, general population $(N=406)$ and depressed patients $(N=106)$. The scale reproduces the results of their authors. It has demonstrated high internal consistency, with Cronbach $\alpha$ ranging between .85 and .93, one-dimensional structure for each scale and the extracted factors explain between $50.9 \%$ and $67.6 \%$ of total variance.

Temporal stability was moderate to high ( $r=.66$ to $r=.92$ ). Patients have significantly higher values on the IES and the EES than controls, supporting the construct validity. The moderate correlations ( $r=.50$ to $r=.64$ ) between EE and the Beck Depression Inventory suggest a convergence between these instruments.

Entrapment Scale has good psychometric characteristics, and must now broaden its application on studies of prospective nature in clinical settings

KEY-WORDS: Entrapment, Evolutionary psychopathology, Psychometric characteristics.

\section{Entrapment - concept, définition et caractéristiques psychométriques de la version portugaise de l'Échelle de Entrapment}

Le rôle des stratégies évolutionnaires dans la psychopathologie est récemment devenu un sujet largement étudié, notamment les mécanismes de lutte et de fuite. Le entrapment désigne l'inhibition de la stratégie de fuite du moment que cela vient psycobiologiquement activé. L'entrapment est souvent lié au stress, anxiété, dépression et suicide dans certains contextes de vie. Ce travail présente les caractéristiques psychométriques de l'échelle d'Entrapment (EE) de qu'a été proposée en origine par Gilbert \& Allan (1998); l'EE est composée par deux échelles, une l'Entrapment Interne (EEI) liée a l'activation de la stratégie de fuite par conséquence d'un état émotionnel adverse, et l'échelle Entrapment Externe (EEE) quand le désir d'échapper s'est stimulé par les événements externes.

L'échelle a été évaluée dans trois échantillons: étudiants $(N=608)$, population générale $(N=406)$ et malades déprimés $(N=106)$. 
L'échelle confirme les résultats obtenus par ses auteurs. Indique une consistance interne élevée, avec un $\alpha$ de Cronbach que varie entre .85 et .93, une structure unidimensionnelle par chaque échelle, et les facteurs extraits expliquent entre $50.9 \%$ et $67.6 \%$ de la variance totale. La stabilité temporelle a été élevée entre $r=.66$ et $r=$ .92. Les patients ont des valeurs d'EEl et EEE augmentées par rapport aux groupes de contrôle, les différences sont significatives et soutenant la validité de construit. Les corrélations modérées obtenues de $r=.50$ jusqu'à $r=.64$ entre l'EE et l'Inventaire de Dépression de Beck suggère une convergence de ces deux instruments. L'Échelle d’Entrapment présente des bonnes caractéristiques psychométriques, mais il faut augmenter son application à des études cliniques prospectives.

MOTS-CLÉS: Entrapment, Psychopathologie evolutionnaire, Caractéristiques psychométriques 